\title{
Combinatorial Unprovability Proofs and Their Model-Theoretic Counterparts
}

\author{
Mojtaba Aghaei and Amir Khamseh
}

\begin{abstract}
For a function $f$ with domain $[X]^{n}$, where $X \subseteq \mathbb{N}$, we say that $H \subseteq X$ is canonical for $f$ if there is a $v \subseteq n$ such that for any $x_{0}, \ldots, x_{n-1}$ and $y_{0}, \ldots, y_{n-1}$ in $H, f\left(x_{0}, \ldots, x_{n-1}\right)=f\left(y_{0}, \ldots, y_{n-1}\right)$ iff $x_{i}=y_{i}$ for all $i \in v$. The canonical Ramsey theorem is the statement that for any $n \in \mathbb{N}$, if $f:[\mathbb{N}]^{n} \rightarrow \mathbb{N}$, then there is an infinite $H \subseteq \mathbb{N}$ canonical for $f$. This paper is concerned with a model-theoretic study of a finite version of the canonical Ramsey theorem with a largeness condition and also a version of the KanamoriMcAloon principle. As a consequence, we produce new indicators for cuts satisfying PA.
\end{abstract}

\section{Introduction}

We begin by recalling some notions and definitions. If $X$ is a set and $n$ is a natural number, then $[X]^{n}$ denotes the collection of subsets of $X$ of cardinality $n$. We will identify a natural number $n$ with the set $\{0, \ldots, n-1\}$. Also we shall use $\mathbb{N}$ to denote the set of natural numbers as well as its cardinality, in the arrow notation below. If $n, k$, and $c$ are either $\mathbb{N}$ or elements of $\mathbb{N}, X \rightarrow(k)_{c}^{n}$ means that whenever $f:[X]^{n} \rightarrow c$ there is $H \subseteq X$ with $|X| \geq k$ such that $f$ is constant on $[H]^{n}$. In this case we say that $H$ is homogeneous for $f$. Using these definitions we can state the infinite Ramsey theorem and its finite version as follows (see [15]).

Theorem 1.1 For any $n, c \in \mathbb{N}, \mathbb{N} \rightarrow(\mathbb{N})_{c}^{n}$.

Theorem 1.2 For any $n, c, k \in \mathbb{N}$, there is an $m \in \mathbb{N}$ such that $m \rightarrow(k)_{c}^{n}$.

Note that the infinite Ramsey theorem is a statement in the second-order language of arithmetic while the finite Ramsey theorem can be formulated in the first-order language of arithmetic and is provable in Peano arithmetic (for more information see

Received December 25, 2010; accepted August 12, 2012

2010 Mathematics Subject Classification: Primary 03F30; Secondary 03B30, 03 C62

Keywords: unprovable statements, Kanamori-McAloon principle, Paris-Harrington principle, canonical Ramsey theorem

(C) 2014 by University of Notre Dame 10.1215/00294527-2420654 
Kaye [9]). Let us recall a variant of Theorem 1.2, the Paris-Harrington principle. A set $H \subseteq \mathbb{N}$ is called relatively large if $|H| \geq \min (H)$. The notation $X \rightarrow_{*}(k)_{c}^{n}$ means that in addition the homogeneous set is relatively large. The Paris-Harrington principle (denoted $\mathrm{PH}$ ) is the following statement:

For any $n, c, k \in \mathbb{N}$, there is an $m \in \mathbb{N}$ such that $m \rightarrow_{*}(k)_{c}^{n}$.

Paris and Harrington [14] showed the following.

\section{Theorem 1.3 PH is not provable in PA.}

Another important PA-unprovable statement was introduced by Kanamori and McAloon [8]. Let $X \rightarrow(k)_{\text {reg }}^{n}$ mean that whenever $f:[X]^{n} \rightarrow \mathbb{N}$ is regressive, that is, $f\left(x_{1}, \ldots, x_{n}\right) \leq x_{1}$ for all $x_{1}<\cdots<x_{n}$ from $X$, then there is $H \subseteq X$ with cardinality $k$ such that for all $x_{1}<\cdots<x_{n}$ from $H, f\left(x_{1}, \ldots, x_{n}\right)$ only depends on $x_{1}$. Such $H$ is called min-homogeneous for $f$. The Kanamori-McAloon principle (denoted $\mathrm{KM}$ ) is the following statement:

For any $n, k \in \mathbb{N}$, there is an $m \in \mathbb{N}$ such that $m \rightarrow(k)_{\text {reg }}^{n}$.

Kanamori and McAloon [8] showed the following.

\section{Theorem 1.4 $\mathrm{KM}$ is not provable in PA.}

It is worth mentioning that $\mathrm{PH}$ and $\mathrm{KM}$ are indeed equivalent and that the equivalence can be proved purely combinatorially. There are many other well-known examples of unprovable statements in the literature. For more information and a discussion and some recent results in the subject we refer the reader to Bovykin [1], [2].

Let us now recall a more general phenomenon, Weiermann's phase-transition program. It is interested in the transition from provability to unprovability of a given assertion by varying a threshold parameter. For the function $F(x)$, let $\mathrm{PH}_{F}$ be the statement similar to $\mathrm{PH}$ when we replace the largeness condition by $|H| \geq F(\min (H))$. Hence the Paris-Harrington theorem implies that $\mathrm{PH}_{i d}$ is not provable in PA. Weiermann [17] extended this theorem by considering some more functions. The binary length $|i|$ of a natural number $i$ is defined by $|i|=\lceil\log (i+1)\rceil$. The $d$-times iterated length function $|\cdot|_{d}$ is defined recursively as $|x|_{0}=x$ and $|x|_{d+1}=\left.|| x\right|_{d} \mid$. Furthermore, let $H_{\alpha}^{-1}$ be the inverse function of the $\alpha$ th member $H_{\alpha}$ of the Hardy hierarchy. Weiermann classified those functions $F$ for which the statement $\mathrm{PH}_{F}$ is not provable in PA as follows (see [17]).

Theorem 1.5 Let $f_{\alpha}(i)=|i|_{H_{\alpha}^{-1}(i)}$. Then for $\alpha \leq \varepsilon_{0}, \mathrm{PH}_{f_{\alpha}}$ is not provable in PA iff $\alpha=\varepsilon_{0}$.

Related results for a fixed dimension can be found in [19].

We now mention the phase transition threshold for the Kanamori-McAloon principle. Let $\mathrm{KM}_{F}$ be the statement similar to $\mathrm{KM}$ when we replace the condition $f\left(x_{1}, \ldots, x_{n}\right) \leq x_{1}$ by $f\left(x_{1}, \ldots, x_{n}\right) \leq F\left(x_{1}\right)$. Hence the Kanamori-McAloon theorem implies that $\mathrm{KM}_{i d}$ is not provable in PA. Moreover, we let $\mathrm{KM}_{F}^{n}$ be the restriction of $\mathrm{KM}_{F}$ to the fixed exponent $n$. Lee found the following results (see [10]).

Theorem 1.6 Let $f_{\alpha}(i)=|i|_{H_{\alpha}^{-1}(i)}$ and $g_{d}(i)=|i|_{d}$.

(1) For $\alpha \leq \varepsilon_{0}, \mathrm{KM}_{f_{\alpha}}$ is not provable in PA iff $\alpha=\varepsilon_{0}$.

(2) $\mathrm{KM}_{f_{\alpha}}^{d+1}$ is provable in I $\Sigma_{d}$ for any $\alpha<\omega_{d+1}$. 
(3) $\mathrm{KM}_{g_{d}}^{d+1}$ is provable in $I \Sigma_{1}$.

(4) $\mathrm{KM}_{g_{d-2}}^{d+1}$ is not provable in $I \Sigma_{d}$.

Let $F_{\alpha}^{-1}$ be the inverse function of the $\alpha$ th member $F_{\alpha}$ of the fast-growing hierarchy. In [5] the gap between (3) and (4) of Theorem 1.6 was closed.



Note that [4] contains some progress concerning regressive functions. It may be worth mentioning that the phase-transition results are not limited to the PH and KM. For a discussion in general we refer the reader to two classical papers [16] and [18] by Weiermann.

The canonical Ramsey theorem [7] is a generalization of Theorem 1.1 and the Kanamori-McAloon principle with no restrictions on the number of colors or regressiveness of coloring. If the function $f$ has domain $[X]^{n}$, where $X \subseteq \mathbb{N}$, we say that $H \subseteq X$ is canonical for $f$ if there is a $v \subseteq n$ satisfying the following condition: for any $x_{0}, \ldots, x_{n-1}$ and $y_{0}, \ldots, y_{n-1}$ in $H, f\left(x_{0}, \ldots, x_{n-1}\right)=f\left(y_{0}, \ldots, y_{n-1}\right)$ iff $x_{i}=y_{i}$ for all $i \in v$. We shall write $v=v(H)$ when $v$ makes $H$ canonical. The canonical Ramsey theorem of Erdös and Rado [7] is the following statement.

Theorem 1.8 For any $n \in \mathbb{N}$, if $f:[\mathbb{N}]^{n} \rightarrow \mathbb{N}$, there is an infinite $H \subseteq \mathbb{N}$ canonical for $f$.

Using the tools of computability theory and reverse mathematics, Mileti [12] studied the effective content of the canonical Ramsey theorem of Erdös and Rado and its relation to the effective content of König's lemma and Ramsey's theorem. He analyzes the complexity of the solutions to computable instances of this problem in terms of the Turing degrees and the arithmetical hierarchy and gives a sharp characterization for the canonical Ramsey theorem for exponent 2.

In [3] Bovykin and Weiermann conduct a model-theoretic investigation of the canonical Ramsey theorem for exponent 2. Their results approximate the logical strength of this principle by the strength of its finite iterations known as density principles. Moreover, they give a characterization of strong cuts in terms of the canonical Ramsey theorem. Finally they notice that over $\mathrm{RCA}_{0}$, the set of first-order consequences of the canonical Ramsey theorem for exponent 2 coincides with PA.

The finite version of the canonical Ramsey theorem with a largeness condition was first considered by Carlucci and Weiermann [6]. The notation $X \stackrel{\text { can }}{\rightarrow}(k)^{n}$ means that whenever $f$ has domain $[X]^{n}$, there is a subset $H \subseteq X$ with cardinality $|H| \geq \max \{\min (H), k\}$ such that $H$ is canonical for $f$. The canonical Ramsey theorem with a largeness condition (denoted ERL) is the following statement:

$$
\text { For any } n, k \in \mathbb{N} \text {, there is an } m \in \mathbb{N} \text { such that } m \stackrel{\text { can }}{\rightarrow}(k)^{n} \text {. }
$$

The Carlucci-Weiermann combinatorial proof which is an adaptation of a proof in [11] shows that the ERL implies the Paris-Harrington principle PH. Let ERL ${ }^{n}(k)$ be the Erdös-Rado numbers by considering a largeness condition, where $n$ is the exponent and $k$ is the size of the canonical set. Similarly, $\mathrm{PH}_{c}^{n}(k)$ will denote the ParisHarrington numbers, where $n$ is the exponent, $c$ is the number of colors, and $k$ is the size of the large homogeneous set. Here is the combinatorial proof of ERL $\rightarrow$ PH copied from the manuscript by Carlucci and Weiermann [6]. It is done by showing that $\mathrm{PH}_{k-n}^{n}(k) \leq N=\operatorname{ERL}^{n}(k)$, for any $k>n$. Let $f:[N]^{n} \rightarrow k-n$ be 
given. By definition, there exists $H \subseteq N$ of size $\max \{k, \min (H)\}$ which is canonical for $f$ with the index set $v$. We shall prove $v=\emptyset$. If $i \in v$, then writing $H$ as $\left\{x_{1}, \ldots, x_{k}, \ldots\right\}$, the values

$$
\begin{aligned}
& f\left(x_{1}, \ldots, x_{i-1}, x_{i}, \ldots, x_{n}\right), \\
& f\left(x_{1}, \ldots, x_{i-1}, x_{i+1}, \ldots, x_{n+1}\right), \\
& \vdots \\
& f\left(x_{1}, \ldots, x_{i-1}, x_{i+(k-n)}, \ldots, x_{k}\right),
\end{aligned}
$$

should all be distinct, which is not the case. Hence $v=\emptyset$, and so $H$ is homogeneous for $f$. The above argument shows that ERL is not provable in PA. Indeed, Carlucci and Weiermann studied the parameterized version of ERL. Let ERL $F_{F}$ be the statement similar to ERL when we replace the largeness condition by $|H| \geq F(\min (H))$. The following result was obtained in [6].

Theorem 1.9 Let $f_{\alpha}(i)=|i|_{H_{\alpha}^{-1}(i)}$.

(1) For $\alpha \leq \varepsilon_{0}$, ERL $_{f_{\alpha}}$ is not provable in PA iff $\alpha=\varepsilon_{0}$.

(2) $\mathrm{ERL}_{\mathrm{Ack}}^{2}-\sqrt[(i)]{i+2}$ is not provable in $I \Sigma_{1}$.

In this paper, we first provide a model-theoretic framework for thinking about the canonical Ramsey theorem and produce a new indicator for models of PA. As a corollary, we get the Carlucci-Weiermann unprovability result. Then in Section 3, we study the model-theoretic treatment of a version of the Kanamori-McAloon principle. Again, as a consequence we have new indicators for models of PA and $I \Sigma_{n}$ for each $n \in \mathbb{N}$.

\section{On the Canonical Ramsey Theorem with a Largeness Condition}

In this section, we study the finite version of ERL from a model-theoretic point of view. To produce an indicator for models of PA, we use the following simple lemma. As usual $[a, b]$ denotes the set $\{a, a+1, \ldots, b-1, b\}$.

Lemma 2.1 Let $[a+m, b] \stackrel{\operatorname{can}_{*}}{\rightarrow}(k)^{n}$, and let $g$ be a function with domain $[[a, b]]^{n}$. Then there exists $H$ which is canonical for $g$ and $|H| \geq \max \{k, \min (H)+m\}$.

Proof Define the function $f$ with domain $[[a+m, b]]^{n}$ by

$$
f\left(x_{1}, \ldots, x_{n}\right)=g\left(x_{1}-m, \ldots, x_{n}-m\right) .
$$

Let $H_{0}$ be canonical for $f$ with $\left|H_{0}\right| \geq \max \left\{k, \min \left(H_{0}\right)\right\}$. Then the set $H=H_{0}-$ $m=\left\{h-m: h \in H_{0}\right\}$ is canonical for $g$ and we have $|H|=\left|H_{0}\right| \geq \min \left(H_{0}\right)=$ $\min (H)+m$.

We now turn to the set $C$ which constitutes a set of diagonal indiscernibles for all $\Delta_{0}$-formulas in the language of arithmetic. Below $r(e)$ is the Ramsey number $R(2 e+1, e+2,3 e+1)$.

Lemma 2.2 Let $M \models I \Sigma_{1}$ be nonstandard. Suppose that for nonstandard elements $e_{1} \leq a_{1}$ in $M$ there exists $b \in M$ such that $\left[a_{1}, b\right] \stackrel{\text { can }}{\rightarrow}\left(2 e_{1}\right)^{e_{1}}$. Then there is $a$ cut $I<M$ such that $e_{1}, a_{1}<I<b$ and $I \models \mathrm{PA}+\neg \forall n, x, z \exists y\left([x, y] \stackrel{\text { can }}{\rightarrow}(z)^{n}\right)$.

Proof By overspill, let $e$ be nonstandard with $r(e)+2 e \leq 2 e_{1}$. Hence $e_{1} \geq 5 e+1>4 e+1$, and if we let $a=a_{1}-(4 e+1)$, then $a \geq e$ and so 
$a$ is nonstandard. Then clearly $[a+4 e+1, b] \stackrel{\text { can }}{\rightarrow}(r(e)+2 e)^{4 e+1}$. Let

$$
\varphi_{1}\left(z, x_{1}, x_{2}, \ldots, x_{e}\right), \ldots, \varphi_{e}\left(z, x_{1}, x_{2}, \ldots, x_{e}\right)
$$

be the first $e \Delta_{0}$-formulas in at most the free variables shown. By Lemma 2.1 and the assumption, let $b \in M$ be minimal such that for every function $g$ with domain $[[a, b]]^{4 e+1}$, there exists a $g$-canonical set $H \subseteq[a, b]$ such that $|H| \geq \max \{r(e)+2 e, \min (H)+4 e+1\}$. We shall build a model of PA between $a$ and $b$.

First define a function $i:[[a, b]]^{2 e+1} \rightarrow e+2$ as follows. For $c<\overline{d_{1}}<\overline{d_{2}}$ in $[a, b]$, put

$$
i\left(c, \overline{d_{1}}, \overline{d_{2}}\right)=\min i \leq e \exists p<c\left(\varphi_{i}\left(p, \overline{d_{1}}\right) \leftrightarrow \varphi_{i}\left(p, \overline{d_{2}}\right)\right),
$$

if such $i$ exists, and put $e+1$ otherwise. Informally, $i\left(c, \overline{d_{1}}, \overline{d_{2}}\right)$ is the first formula with a parameter smaller than $c$ that distinguishes the tuples $\overline{d_{1}}$ and $\overline{d_{2}}$.

Define another function $p:[[a, b]]^{2 e+1} \rightarrow b$ as follows:

$$
p\left(c, \overline{d_{1}}, \overline{d_{2}}\right)=\min p<c\left(\varphi_{i\left(c, \overline{d_{1}}, \overline{d_{2}}\right)}\left(p, \overline{d_{1}}\right) \leftrightarrow \varphi_{i\left(c, \overline{d_{1}}, \overline{d_{2}}\right)}\left(p, \overline{d_{2}}\right)\right),
$$

if $i\left(c, \overline{d_{1}}, \overline{d_{2}}\right) \neq e+1$ and $c$ otherwise. The value $p\left(c, \overline{d_{1}}, \overline{d_{2}}\right)$ is the first parameter $p$ with which $\varphi_{i\left(c, \overline{d_{1}}, \overline{d_{2}}\right)}$ distinguishes $\overline{d_{1}}$ and $\overline{d_{2}}$.

Now let us introduce our coloring $g:[[a, b]]^{4 e+1} \rightarrow b$ as follows:

$$
g\left(c, \overline{d_{1}}, \overline{d_{2}}, \overline{d_{3}}, \overline{d_{4}}\right)= \begin{cases}0 & \text { if } p\left(c, \overline{d_{1}}, \overline{d_{2}}\right)=p\left(c, \overline{d_{3}}, \overline{d_{4}}\right), \\ j+1 & \text { if } j=p\left(c, \overline{d_{1}}, \overline{d_{2}}\right) \neq p\left(c, \overline{d_{3}}, \overline{d_{4}}\right) .\end{cases}
$$

Note that $g\left(c, \overline{d_{1}}, \overline{d_{2}}, \overline{d_{3}}, \overline{d_{4}}\right) \leq c$. Let $H_{0} \subseteq[a, b]$ be a canonical set for $g$ with $v=v\left(H_{0}\right)$ such that $\left|H_{0}\right| \geq r(e)+2 e$ and $\left|H_{0}\right| \geq \min \left(H_{0}\right)+4 e+1$. We now show that either $v=\varnothing$ or $v=\{0\}$. Suppose to the contrary that $v$ contains some $i \neq 0$. Writing $H_{0}$ as $\left\{x_{0}, x_{1}, \ldots, x_{x_{0}+4 e+1}, \ldots\right\}$, we have that

$$
\begin{aligned}
& g\left(x_{0}, \ldots, x_{i-1}, x_{i}, \ldots, x_{4 e}\right), \\
& g\left(x_{0}, \ldots, x_{i-1}, x_{i+1}, \ldots, x_{4 e+1}\right), \\
& \vdots \\
& g\left(x_{0}, \ldots, x_{i-1}, x_{i+x_{0}+1}, \ldots, x_{4 e+x_{0}+1}\right)
\end{aligned}
$$

are as many as $x_{0}+2$ distinct values for $g$, contradicting $g\left(x_{0}, \ldots, x_{4 e}\right) \leq x_{0}$. Hence either $v=\emptyset$ or $v=\{0\}$. Notice that if $v=\emptyset$, then $H_{0}$ is homogeneous for $g$. Also, $\{0\}$-canonicity is stronger than min-homogeneity since it has "if and only if" in the definition, while min-homogeneity has "only if." Let $z_{1}, z_{2}, \ldots, z_{2 e}$ be the last $2 e$ elements of $H_{0}$, and set $H=H_{0}-\left\{z_{1}, z_{2}, \ldots, z_{2 e}\right\}$. Then

$$
|H| \geq \max \left\{r(e), \min \left(H_{0}\right)+2 e+1\right\}=\max \{r(e), \min (H)+2 e+1\} .
$$

Let $j=g\left(c, \overline{d_{1}}, \overline{d_{2}}, z_{1}, \ldots, z_{2 e}\right)$, for $c<\overline{d_{1}}<\overline{d_{2}}$ arbitrary elements of $H$. Then $p\left(c, \overline{d_{1}}, \overline{d_{2}}\right)=p\left(c, z_{1}, \ldots, z_{2 e}\right)$ if $j=0$ and $p\left(c, \overline{d_{1}}, \overline{d_{2}}\right)=j-1$, if $j \neq 0$. Thus $p\left(c, \overline{d_{1}}, \overline{d_{2}}\right)$ is independent of $\overline{d_{1}}$ and $\overline{d_{2}}$ because $j$ is constant or $j$ only depends on $c$ in the cases where $H_{0}$ is homogeneous or min-homogeneous for $g$, respectively.

Since $|H| \geq r(e)$, there is a set $C \subseteq H$ of $3 e+1$ points $c_{0}<c_{1}<\cdots<c_{3 e}$ in $H$ such that for any two $(2 e+1)$-tuples $c<\overline{d_{1}}<\overline{d_{2}}$ and $c^{\prime}<\overline{d_{1}^{\prime}}<\overline{d_{2}^{\prime}}$ in $C$, we have $i\left(c, \overline{d_{1}}, \overline{d_{2}}\right)=i\left(c^{\prime}, \overline{d_{1}^{\prime}}, \overline{d_{2}^{\prime}}\right)$. If the value of $i$ on $[C]^{2 e+1}$ is $i \neq e+1$ taking 
$p=p\left(c_{0}, c_{1}, \ldots, c_{e}\right)=p\left(c_{0}, c_{e+1}, \ldots, c_{2 e}\right)=p\left(c_{0}, c_{2 e+1}, \ldots, c_{3 e}\right)$, we see that the three formulas

$$
\begin{aligned}
& \varphi_{i}\left(p, c_{1}, \ldots, c_{e}\right), \\
& \varphi_{i}\left(p, c_{e+1}, \ldots, c_{2 e}\right), \\
& \varphi_{i}\left(p, c_{2 e+1}, \ldots, c_{3 e}\right)
\end{aligned}
$$

are inequivalent in pairs, which is impossible since we have only two truth values. Hence $i$ has constant value $e+1$ on $[C]^{2 e+1}$. So we can conclude that the set $C^{\prime}=\left\{c_{0}, \ldots, c_{2 e}\right\}$ is our desired set of diagonal indiscernibles; that is, for any standard $\Delta_{0}$-formula $\varphi\left(z, x_{1}, \ldots, x_{n}\right)$, any $c \in C^{\prime}$, any $d_{1}<\cdots<d_{n}$ and $e_{1}<\cdots<e_{n}$ above $c$, and any $p<c$, we have

$$
\varphi\left(p, d_{1}, \ldots, d_{n}\right) \leftrightarrow \varphi\left(p, e_{1}, \ldots, e_{n}\right) .
$$

Now we can repeat the usual argument that the cut $I=\sup _{k \in \mathbb{N}} c_{k}$ is a model of PA. Note that since $e<c_{0} \in I$, then $e \in I$, and so $4 e+1, a+4 e+1$, and $r(e)+2 e$ are in $I$, and hence $e_{1}, a_{1} \in I$. Moreover, since $M \forall \exists y<b\left(\left[a_{1}, y\right] \stackrel{\text { can }}{\rightarrow}\left(2 e_{1}\right)^{e_{1}}\right)$ by $\Delta_{0}$-absoluteness, $I \not \models \exists y<b\left(\left[a_{1}, y\right] \stackrel{\text { can }}{\rightarrow}\left(2 e_{1}\right)^{e_{1}}\right)$, and so

$$
I \models \mathrm{PA}+\neg \forall n, x, z \exists y\left([x, y] \stackrel{\text { can }}{\rightarrow}(z)^{n}\right) .
$$

Using Lemma 2.2, we model-theoretically reproved the Carlucci-Weiermann unprovability result.

Corollary 2.3 We have PA $\nvdash \forall n, x, z \exists y\left([x, y] \stackrel{\text { can }}{\rightarrow}(z)^{n}\right)$.

We then recall a lemma from [9].

Lemma 2.4 (see [9]) Suppose that $e \in M \models \mathrm{PA}, n \neq 0, A \subseteq M$ is a cofinal definable subset of $M$, and $F:[A]^{n} \rightarrow e$ is a definable function. Then there is a definable subset $B \subseteq A$, cofinal in $M$, such that $F$ is constant on $[B]^{n}$.

We shall prove a similar result for the canonical Ramsey theorem by modifying the original proof of Erdös and Rado [7]. Let $A_{i}$ and $B_{i}$ be finite sets. Recall that $A_{1}: A_{2}: \cdots: A_{m}=B_{1}: B_{2}: \cdots: B_{m}$ means that there exists a function $f(x)$ defined for $x \in A_{1} \cup \cdots \cup A_{m}$, which has the following properties. If $x<y$, then $f(x)<f(y)$, and for each $i, 1 \leq i \leq m, B_{i}=\left\{f(x): x \in A_{i}\right\}$.

Lemma 2.5 Suppose that $M \models \mathrm{PA}, 0 \neq n \in \mathbb{N}, X \subseteq M$ is a cofinal definable subset of $M$, and $F$ is a definable function with domain $[X]^{n}$. Then there is a definable subset $H \subseteq X$, cofinal in $M$ and canonical for $F$. In particular, $\mathrm{PA} \vdash \forall x, z \exists y\left([x, y] \stackrel{\text { can }}{\rightarrow}(z)^{n}\right)$.

Proof The proof is by induction on $n$. Let $n=1$. If $F: X \rightarrow e$ for some $e \in M$, then by Lemma 2.4 there is $H \subseteq X$ cofinal in $M$ and homogeneous for $F$. Then $H$ is canonical for $F$ with $v=\varnothing$. If $F(X)$ is cofinal in $M$, let $H=\{x \in X: \forall y<x(F(x) \neq F(y))\}$. Then $H \subseteq M$ is cofinal and $F$ is one-to-one on $H$. So $H$ is canonical for $F$ with $v=\{1\}$. Now let $n>1$. Fix $D_{0}=\{1, \ldots, 2 n\}$, and define the new function $G:[X]^{2 n} \rightarrow \mathcal{P}\left(\left[D_{0}\right]^{n} \times\left[D_{0}\right]^{n}\right)$ as follows. For $A \subseteq X$ with $|A|=2 n$, let $G(A)=\left\{\left(D^{\prime}, D^{\prime \prime}\right): \exists A^{\prime}, A^{\prime \prime} \in[A]^{n}\right.$, $\left.F\left(A^{\prime}\right)=F\left(A^{\prime \prime}\right), A^{\prime}: A^{\prime \prime}: A=D^{\prime}: D^{\prime \prime}: D_{0}\right\}$. Then by Lemma 2.4, there is a definable subset $H \subseteq X$, cofinal in $M$, such that $G$ is constant on $[H]^{2 n}$. We first claim that for $A, B, C, D \in[H]^{n}$ if $F(A)=F(B)$ and $A: B=C: D$, 
then $F(C)=F(D)$. Let $E_{1}$ and $E_{2}$ be elements of $[H]^{2 n}$ with $A \cup B \subseteq E_{1}$, $C \cup D \subseteq E_{2}$, and $A: B: E_{1}=C: D: E_{2}$. Note that $E_{1}$ and $E_{2}$ always exists and $G\left(E_{1}\right)=G\left(E_{2}\right)$. Let $A: B: E_{1}=D^{\prime}: D^{\prime \prime}: D_{0}$. Then since $F(A)=F(B),\left(D^{\prime}, D^{\prime \prime}\right) \in G\left(E_{1}\right)$ and so $\left(D^{\prime}, D^{\prime \prime}\right) \in G\left(E_{2}\right)$. Hence there exist subsets $B^{\prime}, B^{\prime \prime} \subseteq E_{2}$ such that $B^{\prime}: B^{\prime \prime}: E_{2}=D^{\prime}: D^{\prime \prime}: D_{0}=C: D: E_{2}$ and $F\left(B^{\prime}\right)=F\left(B^{\prime \prime}\right)$. Then clearly $F(C)=F(D)$ since $B^{\prime}=C$ and $B^{\prime \prime}=D$, completing the proof of our claim. We now consider two cases as follows.

Case 1. Suppose that $F(A)=F(B)$ only holds if $A=B$. Then $H$ is canonical for $F$ with $v=\{1, \ldots, n\}$ since $F\left(x_{1}, \ldots, x_{n}\right)=F\left(y_{1}, \ldots, y_{n}\right)$ iff $x_{i}=y_{i}$ for all $i \in v$.

Case 2. Let there exist $A_{0}$ and $B_{0}$ in $[H]^{n}$ such that $A_{0} \neq B_{0}$ and $F\left(A_{0}\right)=F\left(B_{0}\right)$. Writing $H$ as $\left\{h_{0}, h_{1}, \ldots\right\}$, let $A_{1}=\left\{h_{2 i}: h_{i} \in A_{0}\right\}$ and $B_{1}=\left\{h_{2 i}: h_{i} \in B_{0}\right\}$. Now choose $h_{i_{0}} \in B_{0}-A_{0}$, and set $B_{2}=B_{1}-\left\{h_{2 i_{0}}\right\} \cup$ $\left\{h_{2 i_{0}+1}\right\}$. Then $F\left(A_{1}\right)=F\left(B_{1}\right)=F\left(B_{2}\right)$ since $A_{0}: B_{0}=A_{1}: B_{1}=A_{1}: B_{2}$ and $F\left(A_{0}\right)=F\left(B_{0}\right)$. Let $H^{\prime}=\left\{h_{2 i}: h_{i} \in H\right\}$. Then define the function $K$ with domain $\left[H^{\prime}\right]^{n-1}$ as follows. For $A \in\left[H^{\prime}\right]^{n-1}$ let $K(A)=F(A \cup\{x\})$, where $A: A \cup\{x\}=B_{0}-\left\{h_{i_{0}}\right\}: B_{0}$. Note that $K$ is well defined, since if $x, y \in H$ and $A: A \cup\{x\}=A: A \cup\{y\}=B_{0}-\left\{h_{i_{0}}\right\}: B_{0}$, then $A \cup\{x\}: A \cup\{y\}=B_{1}: B_{2}$ and so $F(A \cup\{x\})=F(A \cup\{y\})$. In other words,

$$
F\left(x_{1}, \ldots, x_{n}\right)=K\left(x_{1}, \ldots, x_{j-1}, x_{j}, \ldots, x_{n}\right),
$$

where $\left\{x_{1}, \ldots, x_{j-1}, x_{j+1}, \ldots, x_{n}\right\}:\left\{x_{1}, \ldots, x_{n}\right\}=B_{0}-\left\{h_{i_{0}}\right\}: B_{0}$. By the induction hypothesis, there is a subset $H^{\prime \prime} \subseteq H^{\prime}$, cofinal in $H^{\prime}$ and canonical for $K$ with $v=v\left(H^{\prime \prime}\right)$. Then for $x_{1}, \ldots, x_{n}$ and $y_{1}, \ldots, y_{n}$ in $H^{\prime \prime}$,

$$
\begin{aligned}
& F\left(x_{1}, \ldots, x_{n}\right)=F\left(y_{1}, \ldots, y_{n}\right) \\
& \quad \Longleftrightarrow K\left(x_{1}, \ldots, x_{j-1}, x_{j+1}, \ldots, x_{n}\right)=K\left(y_{1}, \ldots, y_{j-1}, y_{j+1}, \ldots, y_{n}\right) \\
& \quad \Longleftrightarrow x_{i}=y_{i}, \quad \text { for each } i \text { such that }(i \in v \wedge i<j) \vee(i>j \wedge i-1 \in v) .
\end{aligned}
$$

So $H^{\prime \prime}$ is canonical for $F$ with $v^{\prime}=\{i: i<j, i \in v\} \cup\{i+1: i>j, i \in v\}$. For the proof of second part, it is enough to follow the proof of [9, Lemma 14.15]. Indeed by the first part, we can find the canonical set $H$ for $F$ with $v=v(H)$. Then

$$
\begin{aligned}
& f_{h_{0}}\left(x_{1}, \ldots, x_{n+1}\right)=f_{h_{0}}\left(y_{1}, \ldots, y_{n+1}\right) \\
& \quad \Longleftrightarrow \quad F\left(x_{1}, \ldots, x_{n}, h_{0}\right)=F\left(y_{1}, \ldots, y_{n}, h_{0}\right) \\
& \Longleftrightarrow \quad x_{i}=y_{i}, \quad \text { for each } i \text { in } v-\{n+1\} .
\end{aligned}
$$

Then $H$ is canonical for $f_{h_{0}}$ with $v^{\prime}=v-\{n+1\}$ and $|H| \geq \min (H)$.

Note that Lemma 2.5 follows from known facts: either from equivalence with the Ramsey theorem that translates homogeneity and canonicity or by Mileti's analysis. Above, we wrote the model-theoretic version. Also note that in a model of PA the canonical sets are definable when you fix dimension and the reader may prove the main statement of Lemma 2.5 via the Ramsey theorem.

Let $a, b \in M \models \mathrm{PA}$, and let $a<I<b$ for $I \models \mathrm{PA}$ and $I<M$. Then by Lemma $2.5, I \models \exists y\left([a, y] \stackrel{\text { can }}{\rightarrow}(2 n)^{n}\right)$ for all $n \in \mathbb{N}$. So

$$
M \models \exists y<b\left([a, y] \stackrel{\text { can }}{\rightarrow}(2 n)^{n}\right),
$$


for all $n \in \mathbb{N}$; hence by overspill,

$$
M \models \exists y<b\left([a, y] \stackrel{\text { can }}{\rightarrow}(2 e)^{e}\right),
$$

for some nonstandard element $e$, and hence

$$
M \models[a, b] \stackrel{\text { can }}{\rightarrow}(2 e)^{e} .
$$

It may be worth mentioning here that there are more colorings encoded in $M$ than are definable in $I$, and in the definition of an indicator we only consider $M$-coded colorings. However since $I$ is closed under exponentiation, $I$ and $M$ encode the same colorings of a segment that is bounded in $I$.

We can now apply Lemma 2.2 to get an indicator for models of PA. Indeed we have the following theorem.

\section{Theorem 2.6 The function}

$$
Y(a, b)=\max e\left([a, b] \stackrel{\text { can }}{\rightarrow}(2 e)^{e}\right)
$$

is an indicator for models of PA. Moreover, the function $v_{a}(n)=$ the least $m$ such that $[a, m] \stackrel{\text { can }}{\rightarrow}(2 n)^{n}$ is not provably total in PA and eventually dominates every provably recursive function of $\mathrm{PA}$.

\section{On a Version of the Kanamori-McAloon Principle}

In this section we will study the model-theoretic treatment of a version of the Kanamori-McAloon principle. We model-theoretically show that this version, denoted by $\mathrm{KM}_{j}$, is not provable in PA. Then we discuss the unprovability results for a fixed exponent. These results will give new indicators for models of PA and $I \Sigma_{n}$, respectively. Let us now introduce the statement $\mathrm{KM}_{j}$ as follows. For all $n$ and $k$ there exists $m$ such that whenever $f$ is $j$-regressive, that is, $f\left(x_{1}, \ldots, x_{n}\right)$ belongs to $\left[x_{j-1}, x_{j}\right]$, then there is a set $H \subseteq m$ with $|H| \geq k$ such that the values of $f$ on $H$ depend only on $x_{1}, \ldots, x_{j}$. We use the notation $m \rightarrow(k)_{j \text {-reg }}^{n}$ for this statement and say that $H$ is $j$-homogeneous. Thus for any $x_{1}<\cdots<x_{n}$ and $y_{1}<\cdots<y_{n}$ in $H$, if for all $i$ with $1 \leq i \leq j, x_{i}=y_{i}$, then $f\left(x_{1}, \ldots, x_{n}\right)=f\left(y_{1}, \ldots, y_{n}\right)$. Another relative statement denoted by $\mathrm{KM}_{j}^{\prime}$ would be the following. For all $n$ and $k$ there exists $m$ such that whenever $f\left(x_{1}, \ldots, x_{n}\right) \leq x_{j}$, then there is a set $H \subseteq m$ with $|H| \geq k$ such that the values of $f$ on $H$ depend only on $x_{1}, \ldots, x_{j}$. Clearly $\mathrm{KM}_{j}^{\prime}$ implies $\mathrm{KM}_{j}$. We now show that $\mathrm{KM}_{j}$ implies KM. Let $f:[[a, b]]^{n} \rightarrow b$ be regressive. Then define the function $g:[[a, b]]^{n+j} \rightarrow b$ by

$$
g\left(x_{1}, \ldots, x_{n+j}\right)= \begin{cases}f & \text { if } f \geq x_{j-1}, \\ x_{j-1}+f & \text { if } f<x_{j-1} \& 2 x_{j-1} \leq x_{j}, \\ x_{j} & \text { otherwise, }\end{cases}
$$

where $f=f\left(x_{j}, x_{j+1}, \ldots, x_{n+j}\right) \leq x_{j}$. Then $g\left(x_{1}, \ldots, x_{n+j}\right) \in\left[x_{j-1}, x_{j}\right]$, and so there exists $H_{0} \subseteq[a, b]$ such that $\left|H_{0}\right| \geq k+j-1$ and the values of $g$ on $H_{0}$ depend on $x_{1}, \ldots, x_{j}$. We can assume that for $x<y$ both in $H_{0}$ we have $2 x<y$ (see Remark 3.2 and Lemma 3.3). Let $z_{1}<\cdots<z_{j-1}$ be the first $j-1$ elements of $H_{0}$. Let $H_{1}=H_{0}-\left\{z_{1}, \ldots, z_{j-1}\right\}$. Then $\left|H_{1}\right| \geq k$ and for any $x_{1}<x_{2}<\cdots<x_{n}$ and $x_{1}<y_{2}<\cdots<y_{n}$ in $H_{1}$, we have

$$
f\left(x_{1}, x_{2}, \ldots, x_{n}\right)=f\left(x_{1}, y_{2}, \ldots, y_{n}\right)
$$


since $g\left(z_{1}, \ldots, z_{j-1}, x_{1}, x_{2}, \ldots, x_{n}\right)=g\left(z_{1}, \ldots, z_{j-1}, x_{1}, y_{2}, \ldots, y_{n}\right)$, that is, $H_{1}$ is min-homogeneous for $f$.

Note here that for a $j$-regressive function $f$ we cannot guarantee the existence of a set $H$ such that the values of $f$ on $H$ depend only on $x_{j-1}$ and $x_{j}$. To see this, define the function $f:[\mathbb{N}]^{4} \rightarrow \mathbb{N}$ by $f\left(x_{1}, x_{2}, x_{3}, x_{4}\right)=x_{2}+\left[\frac{x_{1}}{x_{1}+1}\left(x_{3}-x_{2}\right)\right]$. Then clearly $f\left(x_{1}, x_{2}, x_{3}, x_{4}\right) \in\left[x_{2}, x_{3}\right]$. Let $H=\{a, b, c, \ldots, d, \ldots, e, \ldots\} \subseteq \mathbb{N}$ be infinite, and let $(d-c)\left(\frac{b}{b+1}-\frac{a}{a+1}\right)>1$. Then $\left[\frac{a}{a+1}(d-c)\right]<\left[\frac{b}{b+1}(d-c)\right]$, and so $f(a, c, d, e)<f(b, c, d, e)$.

On the other hand, suppose that $f\left(x_{1}, \ldots, x_{n}\right) \in\left[x_{i}, x_{j}\right]$ for some $i, j$ with $1 \leq i<j \leq n$. Then the values of $f$ on a set $H$ cannot be independent of any $x_{k}$ with $i \leq k \leq j$; a simple counterexample is the function $f\left(x_{1}, \ldots, x_{n}\right)=x_{k}$. Similarly, for a function $f$ with $f\left(x_{1}, \ldots, x_{n}\right) \leq x_{j}$ the values of $f$ cannot be independent of each $x_{k}$ with $1 \leq k \leq j$. For the infinite case $\mathrm{KM}_{j}$ and $\mathrm{KM}_{j}^{\prime}$ are the weakest and the strongest cases, respectively. On the surface the modifications of KM look too similar to KM itself. Actually, since there is dependence on $j$-coordinates, the relation is not that close and they are different enough to study here.

Let us now recall the regressive functions $\eta_{1}, \eta_{2}$, and $\eta_{3}$ from [8]. Let $\eta_{1}(x, y)$ be 0 if $x+x \leq y$ and be $y-x$ otherwise. Also define $\eta_{2}(x, y)=0$ if $x . x \leq y$ and $\eta_{2}(x, y)=u$ otherwise where $u . x \leq y<(u+1) \cdot x$. Finally, $\eta_{3}(x, y)=0$ if $x^{x} \leq y$ and $\eta_{3}(x, y)=v$ otherwise where $x^{v} \leq y<x^{v+1}$. Now we have the following lemma.

Lemma 3.1 (see [8]) If $\bar{H}$ is min-homogeneous for $\eta_{1}, \eta_{2}$, and $\eta_{3}$, then $\bar{H}-$ (the last three elements of $\bar{H})$ has the property that $x<y$ both in $H$ implies $x^{x} \leq y$.

Remark 3.2 For a given function $t$ let $\mathrm{KM}_{t}$ be the following statement. For all $k$ and $n$ there exists $m$ such that $m \rightarrow_{t}(k)_{\text {reg }}^{n}$, where $m \rightarrow_{t}(k)_{\text {reg }}^{n}$ means that whenever $f:[m]^{n} \rightarrow m$ is regressive, then there is $H$ min-homogeneous for $f$ such that $|H| \geq k$ and $x<y$ both in $H$ implies that $t(x) \leq y$. It is proved in [8] that if $t(x)=x^{x}$, then $\mathrm{KM}$ implies $\mathrm{KM}_{t}$. Lemma 3.3 shows that a similar result holds for $\mathrm{KM}_{j}$.

Lemma 3.3 Let $t(x)=x^{x}$. Then $I \Sigma_{1} \vdash \mathrm{KM}_{j} \rightarrow\left(\mathrm{KM}_{j}\right)_{t}$.

Proof Let $M \models I \Sigma_{1}$. First define the function $g:[M]^{2} \rightarrow M$ by $g(x, y)=0$ if $\eta_{l}(x, y)=0$ for each $l, 1 \leq l \leq 3$, and $g(x, y)=l$ otherwise where $l$ is the least with $\eta_{l}(x, y) \neq 0$. Let $[a, b] \rightarrow(R(2,4, k+3))_{j \text {-reg }}^{n+j}$. For the $j$-regressive function $f:[[a, b]]^{n+j} \rightarrow b$ let us introduce the coloring $h:[[a, b]]^{n+j} \rightarrow b$ by

$$
h\left(x_{1}, \ldots, x_{n+j}\right)= \begin{cases}x_{j} & \text { if } g\left(x_{j}, x_{j+1}\right)>0 \\ f\left(x_{1}, \ldots, x_{n+j}\right) & \text { if } g\left(x_{j}, x_{j+1}\right)=0\end{cases}
$$

By $\mathrm{KM}_{j}^{n+j}$, there exists $H_{0} \subseteq[a, b]$ with $\left|H_{0}\right| \geq k+3$ such that $H_{0}$ is $j$-homogeneous for $h$ and homogeneous for $g$. Let $H_{1}=H_{0}-\left\{z_{1}, z_{2}, z_{3}\right\}$, where $z_{1}<z_{2}<z_{3}$ are the last three elements of $H_{0}$. Clearly if $g$ on $H_{1}$ were constantly $l>0$, then we can get a contradiction (see [8, proof of Lemma 4.2]). Hence $h=f$ on $H_{1}$, and by Lemma 3.3 for $x<y$ both in $H_{1}$ we have $x^{x} \leq y$.

Lemma 3.4 Let $j \in \mathbb{N}$ and $M \models I \Sigma_{1}$ be nonstandard. Suppose that for nonstandard elements $a, e, n$, and $k$ in $M$ and the function $t(x)=2 x$ there exists $b \in M$ 
such that $[a, b] \rightarrow_{t}(w)_{j \text {-reg }}^{2 n+j}$, where $w$ is the Ramsey number $R(2 n+1, e+2, d)$ with $d=\max \{k+n, r+j\}$ and $r=R(2 n, 2,3 n)$. Then there is a cut $I<M$ such that $a<I<b$ and $I \models \mathrm{PA}+\neg \forall n, z \exists y\left([a, y] \rightarrow(z)_{j \text {-reg }}^{n}\right)$.

Proof Let $b$ be minimal such that $[a, b] \rightarrow_{t}(w)_{j \text {-reg }}^{2 n+j}$, where $w$ is the Ramsey number $R(2 n+1, e+2, d)$ with $d=\max \{k+n, r+j\}$ and $r=R(2 n, 2,3 n)$. Let

$$
\varphi_{0}\left(z, x_{1}, x_{2}, \ldots, x_{n}\right), \ldots, \varphi_{e}\left(z, x_{1}, x_{2}, \ldots, x_{n}\right)
$$

be the first $\Delta_{0}$-formulas in the language of arithmetic in at most the free variables shown. We shall prove there exists $H$ with $|H| \geq k$ which constitutes a set of diagonal indiscernibles for these formulas; that is, for $c_{0}<c_{1}<\cdots<c_{n}$ and $c_{0}<d_{1}<\cdots<d_{n}$ in $H$, any $p<c_{0}$, and all $i \leq e$, we have

$$
\varphi_{i}\left(p, c_{1}, \ldots, c_{n}\right) \leftrightarrow \varphi_{i}\left(p, d_{1}, \ldots, d_{n}\right) .
$$

First define the function $i:[a, b]^{2 n+1} \rightarrow e+2$ as follows.

For $x_{0}<x_{1}<\cdots<x_{2 n}$ in $[a, b]$, let

$$
\begin{aligned}
& i\left(x_{0}, x_{1}, \ldots, x_{2 n}\right) \\
& \quad=\min i \leq e \exists p<x_{0}\left(\varphi_{i}\left(p, x_{1}, \ldots, x_{n}\right) \leftrightarrow \varphi_{i}\left(p, x_{n+1}, \ldots, x_{2 n}\right)\right)
\end{aligned}
$$

if such $i$ exists, and $e+1$ otherwise. Also define the function $p:[a, b]^{2 n+1} \rightarrow b$ by

$$
\begin{aligned}
& p\left(x_{0}, x_{1}, \ldots, x_{2 n}\right) \\
& \quad=\min p<x_{0}\left(\varphi_{i}\left(p, x_{1}, \ldots, x_{n}\right) \leftrightarrow \varphi_{i}\left(p, x_{n+1}, \ldots, x_{2 n}\right)\right),
\end{aligned}
$$

if $i \leq e$ and by $x_{0}$ otherwise, where $i=i\left(x_{0}, x_{1}, \ldots, x_{2 n}\right)$. Then define the function $f:[a, b]^{2 n+j} \rightarrow b$ as follows.

For $y_{1}<y_{2}<\cdots<y_{j-1}<x_{0}<x_{1}<\cdots<x_{2 n}$ in $[a, b]$, let

$$
f\left(y_{1}, y_{2}, \ldots, y_{j-1}, x_{0}, x_{1}, \ldots, x_{2 n}\right)= \begin{cases}p & \text { if } y_{j-1} \leq p, \\ y_{j-1}+p & \text { if } p<y_{j-1} \& 2 y_{j-1} \leq x_{0}, \\ x_{0} & \text { otherwise, }\end{cases}
$$

where $p=p\left(x_{0}, x_{1}, \ldots, x_{2 n}\right)$. Since $[a, b] \rightarrow_{t}(w)_{j \text {-reg }}^{2 n+j}$, there exists $H_{0}$ which is $j$-homogeneous for $f$ with $H_{0} \subseteq[a, b],\left|H_{0}\right| \geq w$, and for each $x, y \in H_{0}$ if $x<y$, then $2 x<y$. By the assumption on $w$, there is $H_{1} \subseteq H_{0}$ with $\left|H_{1}\right| \geq k+n$ and $\left|H_{1}\right| \geq r+j$ such that the function $i$ has constant value $i$ on $\left[H_{1}\right]^{2 n+1}$.

First suppose that $i=e+1$. Let $H_{2}=H_{1}-\left\{z_{1}, \ldots, z_{n}\right\}$, where $z_{1}<\cdots<z_{n}$ are the last $n$ elements of $H_{1}$. Then $\left|H_{2}\right| \geq k$ and for $c_{0}<c_{1}<\cdots<c_{n}$ and $c_{0}<d_{1}<\cdots<d_{n}$ elements of $H_{2}$, we have

$$
\begin{aligned}
e+1 & =i\left(c_{0}, c_{1}, \ldots, c_{n}, z_{1}, \ldots, z_{n}\right) \\
& =i\left(c_{0}, d_{1}, \ldots, d_{n}, z_{1}, \ldots, z_{n}\right) .
\end{aligned}
$$

Hence for any $p<c_{0}$, and all $i \leq e$,

$$
\varphi_{i}\left(p, c_{1}, \ldots, c_{n}\right) \leftrightarrow \varphi_{i}\left(p, z_{1}, \ldots, z_{n}\right) \leftrightarrow \varphi_{i}\left(p, d_{1}, \ldots, d_{n}\right) .
$$

Now suppose that $i \leq e$, and let $y_{1}<\cdots<y_{j-1}<x_{0}$ be the first $j$ elements of $H_{1}$. Let $H_{2}=H_{1}-\left\{y_{1}, \ldots, y_{j-1}, x_{0}\right\}$. Then $\left|H_{2}\right| \geq r$. Moreover, since $x_{0} \geq 2 y_{j-1}$ we see that for $x_{1}<\cdots<x_{2 n}$ in $H_{2}$ the value of $f\left(y_{1}, y_{2}, \ldots, y_{j-1}, x_{0}, x_{1}, \ldots, x_{2 n}\right)$ is equal to either $p\left(x_{0}, x_{1}, \ldots, x_{2 n}\right)$ or $p\left(x_{0}, x_{1}, \ldots, x_{2 n}\right)+y_{j-1}$. 
Now define the function $g:\left[H_{2}\right]^{2 n} \rightarrow 2$ as $g\left(x_{1}, \ldots, x_{n}, x_{n+1}, \ldots, x_{2 n}\right)=0$ if $f\left(y_{1}, y_{2}, \ldots, y_{j-1}, x_{0}, x_{1}, \ldots, x_{2 n}\right)=p$ and 1 otherwise. Then since $\left|H_{2}\right| \geq r$, there exists $H_{3} \subseteq H_{2}$ such that $\left|H_{3}\right| \geq 3 n$ and $H_{3}$ is homogeneous for $g$.

Let $x_{1}<\cdots<x_{n}<x_{n+1}<\cdots<x_{2 n}<x_{2 n+1}<\cdots<x_{3 n}$ be elements of $H_{3}$. Then

$$
\begin{aligned}
& f\left(y_{1}, y_{2}, \ldots, y_{j-1}, x_{0}, x_{1}, \ldots, x_{2 n}\right) \\
& \quad=f\left(y_{1}, y_{2}, \ldots, y_{j-1}, x_{0}, x_{1}, \ldots, x_{n}, x_{2 n+1}, \ldots, x_{3 n}\right) \\
& \quad=f\left(y_{1}, y_{2}, \ldots, y_{j-1}, x_{0}, x_{n+1}, \ldots, x_{2 n}, x_{2 n+1}, \ldots, x_{3 n}\right) .
\end{aligned}
$$

The common value above is always $p$ or always $p+y_{j-1}$, and in both cases we get

$$
\begin{aligned}
p & =p\left(x_{0}, x_{1}, \ldots, x_{2 n}\right) \\
& =p\left(x_{0}, x_{1}, \ldots, x_{n}, x_{2 n+1}, \ldots, x_{3 n}\right) \\
& =p\left(x_{0}, x_{n+1}, \ldots, x_{2 n}, x_{2 n+1}, \ldots, x_{3 n}\right) .
\end{aligned}
$$

Thus for this $p$ we see that the three formulas

$$
\varphi_{i}\left(p, x_{1}, \ldots, x_{n}\right), \quad \varphi_{i}\left(p, x_{n+1}, \ldots, x_{2 n}\right), \quad \varphi_{i}\left(p, x_{2 n+1}, \ldots, x_{3 n}\right)
$$

are inequivalent in pairs, which is impossible. This contradiction shows that $i=e+1$, yielding the desired set of diagonal indiscernibles. Again we can repeat the usual argument that leads to the cut $I<M$ with $a<I<b$ and $I \models \mathrm{PA}+\neg \forall n, z \exists y\left([a, y] \rightarrow(z)_{j \text {-reg }}^{n}\right)$.

Let $a, b \in M \models \mathrm{PA}$ and $a<I<b$ for $I \models \mathrm{PA}$ and $I<M$. Then by Theorem 3.9,

$$
I \models \exists y\left([a, y] \rightarrow(2 n)_{j-\text { reg }}^{n+j}\right)
$$

for all $n \in \mathbb{N}$. So

$$
M \models \exists y<b\left([a, y] \rightarrow(2 n)_{j \text {-reg }}^{n+j}\right)
$$

for all $n \in \mathbb{N}$, hence by overspill

$$
M \models \exists y<b\left([a, y] \rightarrow(2 c)_{j \text {-reg }}^{c}\right)
$$

for some nonstandard element $c$, and hence

$$
[a, b] \rightarrow(2 c)_{j \text {-reg }}^{c} \text {. }
$$

Now suppose that $c>\mathbb{N}$ and $M \models[a, b] \rightarrow(2 c)_{j \text {-reg. }}^{c}$ By an overspill argument we can see that $R(2 e+1, e+2, d)<2 c$ for some nonstandard $e$, where $d=\max \{k+e, r+j\}$ and $r=R(2 e, 2,3 e)$. Then $[a, b] \rightarrow(w)_{j \text {-reg }}^{2 e+j}$, and so by Lemma 3.4, there exists $I \models$ PA with $I<M$ and $a<I<b$. Summarizing, we have an indicator for models of PA.

Theorem 3.5 Let $j \in \mathbb{N}$. Then $\mathrm{KM}_{j}$ is not provable in $\mathrm{PA}$, and the function

$$
Y(a, b)=\max c\left([a, b] \rightarrow(2 c)_{j \text {-reg }}^{c}\right)
$$

is an indicator for models of PA. Moreover, the function $v(n)=$ the least $m$ such that $m \rightarrow(2 n)_{j \text {-reg }}^{n}$ is not provably total in PA and eventually dominates every provably recursive function of $\mathrm{PA}$.

We now turn our attention to $\mathrm{KM}_{j}^{n}$, the restriction of $\mathrm{KM}_{j}$ to the fixed exponent $n$. By modifying some proofs in [8], we show that one can get the set of diagonal indiscernibles as in Lemma 3.4 by considering $\mathrm{KM}_{j}^{n+j}$. 
Lemma 3.6 Let $j \in \mathbb{N}$ and $M \models I \Sigma_{1}$ be nonstandard. Let $n \in \mathbb{N}$, and suppose that for nonstandard elements $a, k$ in $M$ and the function $t(x)=x^{x}$ there exists $b \in M$ such that $[a, b] \rightarrow_{t}(k)_{j \text {-reg. }}^{n+j}$. Then there is a cut $I<M$ such that $a<I<b$ and $I \models I \Sigma_{n}+\neg \forall z \exists y\left([a, y] \rightarrow_{t}(z)_{j \text {-reg }}^{n+j}\right)$.

Proof Since $2^{m+1}<a$ for each $m \in \mathbb{N}$, by overspill we have $2^{e+1}<a$ for some $e \in M-\mathbb{N}$. We shall show that for $\Delta_{0}$-formulas $\varphi_{0}, \ldots, \varphi_{e}$ in the language of arithmetic with at most $n+1$ free variables, there exists a set $H \subseteq M$ with $|H| \geq k$ which constitutes a set of diagonal indiscernibles for these formulas. First, define the function $q: M \rightarrow M$ by $q(x)=$ the largest $d$ such that $2^{(e+1) \cdot d} \leq x$. Let $b$ be minimal such that $[a, b] \rightarrow_{t}(k)_{j \text {-reg }}^{n+j}$. Then define the function $f:[[a, b]]^{n+j} \rightarrow b$ by

$$
\begin{aligned}
& f\left(y_{1}, \ldots, y_{j-1}, x_{0}, \ldots, x_{n}\right) \\
& \quad= \begin{cases}\delta:=\left\langle\delta_{i p}: i \leq e, p<q\left(x_{0}\right)\right\rangle & \text { if } y_{j-1} \leq \delta<x_{0}, \\
\delta+y_{j-1} & \text { if } \delta<y_{j-1} \leq x_{0} / 2, \\
x_{0} & \text { otherwise, }\end{cases}
\end{aligned}
$$

where $\delta_{i p}\left(x_{1}, \ldots, x_{n}\right)=0$ if $\varphi_{i}\left(p, x_{1}, \ldots, x_{n}\right)$ is true and $\delta_{i p}\left(x_{1}, \ldots, x_{n}\right)=1$ otherwise.

By the assumption there exists $H_{1} j$-homogeneous for $f$ such that $\left|H_{1}\right| \geq$ $R(n, 2,2 k)+j$ and for $x<y$ both in $H_{1}$ we have $x^{x} \leq y$. Then either $f=\delta$ or $f=\delta+y_{j-1}$ on $H_{1}$. Moreover, $x<y$ both in $H_{1}$ implies $x \leq q(y)$ since $2^{(e+1) x}=\left(2^{e+1}\right)^{x}<a^{x} \leq x^{x} \leq y$. Now set $H_{2}=H_{1}-\left\{y_{1}, \ldots, y_{j-1}\right\}$, where $y_{1}<\cdots<y_{j-1}$ are the first $j-1$ elements of $H_{2}$, and define the function $g:\left[H_{2}\right]^{n+1} \rightarrow 2$ as follows: $g\left(x_{0}, x_{1}, \ldots, x_{n}\right)$ is 0 if $f\left(y_{1}, \ldots, y_{j-1}, x_{0}, \ldots\right.$, $\left.x_{n}\right)=\delta$ and is 1 if $f\left(y_{1}, \ldots, y_{j-1}, x_{0}, \ldots, x_{n}\right)=\delta+y_{j-1}$. Then by assumption on $H_{2}$, there exists $H_{3} \subseteq H_{2}$ with $H_{3} \geq 2 k$ such that $g$ is constant on $H_{3}$. Since for $c_{0}<c_{1}<\cdots<c_{n}$ and $c_{0}<d_{1}<\cdots<d_{n}$ in $H_{3}$,

$$
\delta\left(c_{0}, c_{1}, \ldots, c_{n}\right)=\delta\left(c_{0}, d_{1}, \ldots, d_{n}\right),
$$

we get

$$
f\left(y_{1}, \ldots, y_{j-1}, c_{0}, c_{1}, \ldots, c_{n}\right)=f\left(y_{1}, \ldots, y_{j-1}, c_{0}, d_{1}, \ldots, d_{n}\right),
$$

and so $\delta_{i p}\left(c_{1}, \ldots, c_{n}\right)=\delta_{i p}\left(d_{1}, \ldots, d_{n}\right)$ for each $i \leq e$ and $p<q\left(c_{0}\right)$. Writing $H_{3}$ as $\left\{h_{1}, h_{2}, \ldots, h_{2 k}\right\}$, the subset $\left\{h_{2}, h_{4}, \ldots, h_{2 k}\right\}$ constitutes the set of $k$ diagonal indiscernibles for formulas $\varphi_{0}, \ldots, \varphi_{e}$, as required. It is not hard now to find the cut $I<M$ with $a<I<b$ and $I \models I \Sigma_{n}+\neg \forall z \exists y\left([a, y] \rightarrow_{t}(z)_{j \text {-reg }}^{n+j}\right)$.

Using Lemmas 3.3 and 3.6 we have the following.

Theorem 3.7 We have I $\Sigma_{n} \nvdash \forall x, z \exists y\left([x, y] \rightarrow(z)_{j \text {-reg }}^{n+j}\right)$. Moreover, in terms of the indicator theory of Paris and Kirby, the function

$$
Y(a, b)=\max c\left([a, b] \rightarrow(c)_{j \text {-reg }}^{n+j}\right)
$$

is an indicator for models of $I \Sigma_{n}$.

Lemma 3.8 Let $A=\left\{a_{1}, \ldots, a_{m}\right\}$, where $a_{1}<a_{2}<\cdots<a_{m}<a$. Then the number of $j$-regressive functions on $[A]^{n-1}$ is at most $a^{2^{2 a}}$.

Proof The proof is straightforward. 
By Lemma 3.8 and using the notion of arboricity and prehomogeneous sets of Mills [13], the following is immediate from the proof of [13, Lemma 3.5]. Suppose that $c, n \geq 1, f$ with domain $[A]^{n+j}$ is $j$-regressive, and $A$ is $n$-fold $\left(c-x^{2^{2 x}}\right)$-arboreal with $\min (A)>0$. Then there is an $H \subseteq A$ such that $H$ is prehomogeneous for $f$ and $H-\{\max (H)\}$ is $(n-1)$-fold $\left(c-x^{2^{2 x}}\right)$-arboreal. We can now proceed as in [8, Proposition 4.1] to establish that $\mathrm{PH}^{n+1}$ implies $\mathrm{KM}_{j}^{n+j}$ and also $\mathrm{KM}_{j}^{\prime n+j}$. Moreover, it is well known [8] that both of $\mathrm{PH}^{n+1}$ and $\mathrm{KM}^{n+1}$ are equivalent to $1-\operatorname{Con}\left(I \Sigma_{n}\right)$. Since $I \Sigma_{n+1} \vdash 1-\operatorname{Con}\left(I \Sigma_{n}\right)$, we have the following theorem.

Theorem 3.9 We have $I \Sigma_{n+1} \vdash \forall x, z \exists y\left([x, y] \rightarrow(z)_{j \text {-reg }}^{n+j}\right)$.

Theorem 3.9 together with Theorem 3.7 completely gives the strength of $\mathrm{KM}_{j}$ for a fixed exponent, and so we can summarize the results of this section as follows.

Corollary 3.10 The following are equivalent in $I \Sigma_{1}$ :

(a) $\mathrm{PH}^{n+1}$,

(b) $\mathrm{KM}^{n+1}$,

(c) $\mathrm{KM}_{j}^{n+j}$,

(d) $\mathrm{KM}_{j}^{\prime n+j}$,

(e) $1-\operatorname{Con}\left(I \Sigma_{n}\right)$.

\section{References}

[1] Bovykin, A., "Several proofs of PA-unprovability," pp. 29-43 in Logic and Its Applications, vol. 380 of Contemporary Mathematics, American Mathematical Society, Providence, 2005. MR 2167572. DOI 10.1090/conm/380/07105. 232

[2] Bovykin, A., "Brief introduction to unprovability," pp. 38-64 in Logic Colloquium 2006, Lecture Notes in Logic, Association for Symbolic Logic, Chicago, 2009. MR 2562547. DOI 10.1017/CBO9780511605321.004. 232

[3] Bovykin, A., and A. Weiermann, "The strength of infinitary Ramseyan statements can be accessed by their densities," to appear in Annals of Pure and Applied Logic. 233

[4] Carlucci, L., G. Lee, and A. Weiermann, "Sharp thresholds for hypergraph regressive Ramsey numbers," Journal of Combinatorial Theory, Series A, vol. 118 (2011), pp. 55885. MR 2739504. DOI 10.1016/j.jcta.2010.08.004. 233

[5] Carlucci, L., G. Lee, and A. Weiermann, "Classifying the phase transition threshold for regressive Ramsey functions," preprint, 2006.

[6] Carlucci, L., and A. Weiermann, "Classifying the phase transition for canonical Ramsey functions," preprint, 2010. 233, 234

[7] Erdös, P., and R. Rado, “A combinatorial theorem," Journal of the London Mathematical Society, vol. 25 (1950), pp. 249-55. MR 0037886. 233, 236

[8] Kanamori, A., and K. McAloon, "On Gödel incompleteness and finite combinatorics," Annals of Pure and Applied Logic, vol. 33 (1987), pp. 23-41. MR 0870685. DOI 10.1016/0168-0072(87)90074-1. 232, 239, 241, 243

[9] Kaye, R., Models of Peano Arithmetic, vol. 15 of Oxford Logic Guides, Oxford University Press, New York, 1991. MR 1098499. 232, 236, 237

[10] Lee, G., "Phase transitions in axiomatic thought," Ph.D. dissertation, University of Münster, Münster, Germany, 2005.

[11] Lefmann, H., and V. Rödl, "On canonical Ramsey numbers for complete graphs versus paths," Journal of Combinatorial Theory, Series B, vol. 58 (1993), pp. 1-13. MR 1214886. DOI 10.1006/jctb.1993.1025. 233 
[12] Mileti, J. R., “The canonical Ramsey theorem and computability theory," Transactions of the American Mathematical Society, vol. 360 (2008), pp. 1309-40. MR 2357697. DOI 10.1090/S0002-9947-07-04390-5. 233

[13] Mills, G., "A tree analysis of unprovable combinatorial statements," pp. 248-311 in Model Theory of Algebra and Arithmetic (Karpacz, Poland, 1978), vol. 834 of Lecture Notes in Mathematics, Springer, Berlin, 1980. MR 0606790. 243

[14] Paris, J., and L. Harrington, "A mathematical incompleteness in Peano arithmetic," pp. 1133-42 in Handbook of Mathematical Logic, edited by J. Barwise, vol. 90 of Studies in Logic and the Foundations of Mathematics, North-Holland, Amsterdam, 1977. MR 0457132. 232

[15] Ramsey, F. P., "On a problem of formal logic," Proceedings of the London Mathematical Society, vol. 30 (1930), pp. 264-86. MR 1576401. DOI 10.1112/plms/s2-30.1.264. 231

[16] Weiermann, A., "An application of graphical enumeration to PA," Journal of Symbolic Logic, vol. 68 (2003), pp. 5-16. MR 1959309. DOI 10.2178/jsl/1045861503. 233

[17] Weiermann, A., "A classification of rapidly growing Ramsey functions," Proceedings of the American Mathematical Society, vol. 132 (2004), pp. 553-61. MR 2022381. DOI 10.1090/S0002-9939-03-07086-2. 232

[18] Weiermann, A., "Analytic combinatorics, proof-theoretic ordinals, and phase transitions for independence results, Annals of Pure and Applied Logic, vol. 136 (2005), pp. 189218. MR 2162854. DOI 10.1016/j.apal.2005.05.012. 233

[19] Weiermann, A., and W. Van Hoof, "Sharp phase transition thresholds for the Paris Harrington Ramsey numbers for a fixed dimension," Proceedings of the American Mathematical Society, vol. 140 (2012), pp. 2913-27. MR 2910777. DOI 10.1090/S0002-99392011-11121-3. 232

\title{
Acknowledgments
}

This research was supported in part by a grant (no. 92030031) from the Institute for Research in Fundamental Sciences (IPM). The authors wish to thank the referee for many rounds of reading the paper, for several helpful comments, and for useful suggestions which improved the paper. Also, we are greatly indebted to A. Bovykin for his encouragements, supports, and many stimulating conversations. Finally, we would like to express our deep gratitude to L. Carlucci and A. Weiermann for useful comments and for giving us permission to include parts of their unpublished manuscript.

\author{
Aghaei \\ Department of Mathematical Sciences \\ Isfahan University of Technology \\ 84156-83111 Isafan \\ Iran \\ aghaei@cc.iut.ac.ir \\ Khamseh \\ Department of Mathematics \\ Kharazmi University \\ 15719-14911 Tehran \\ Iran \\ and \\ School of Mathematics \\ Institute for Research in Fundamental Sciences (IPM) \\ PO Box 19395-5746 \\ Tehran \\ Iran \\ khamseh@khu.ac.ir
}

